\title{
Artroplastia total do quadril não cimentada em pacientes com artrite reumatóide*
}

\author{
Uncemented total hip arthroplasty in \\ patients with rheumatoid arthritis
}

\author{
Bruno tavares Rabello ${ }^{1}$, Fernando Pina Cabral ${ }^{2}$, Emílio Freitas ${ }^{3}$, Jorge Penedo ${ }^{3}$, \\ Marco Bernardo Cury33, Eduardo Regado Rinaldi³ ${ }^{3}$ Lourenço Peixoto ${ }^{4}$
}

\section{RESUMO}

Objetivo: Analisar os resultados clínico-funcional e radiográfico das artroplastias totais do quadril não cimentadas em pacientes com artrite reumatóide. Métodos: Estudo retrospectivo de 24 pacientes (28 quadris) portadores de artrite reumatóide submetidos à artroplastia total do quadril, utilizando-se a prótese femoral não cimentada Secur Fit Osteonics ${ }^{\circledR}$, com seguimento mínimo de dois anos. Clinicamente, os pacientes foram avaliados utilizando os critérios de Merle D'Aubigné, e os critérios de Engh, na avaliação radiográfica. Resultados: $O$ tempo médio de seguimento foi de 5,6 anos (dois anos a oito anos e 11 meses). Clinicamente, resulta-

* Trabalho realizado no Instituto Nacional de Traumatologia e Ortopedia - INTO - Rio de Janeiro (RJ), Brasil.

1. Médico Ortopedista; Residente do 4o ano do Grupo de Quadril do Instituto Nacional de Traumatologia e Ortopedia - INTO Rio de Janeiro (RJ), Brasil.

2. Médico Ortopedista, Chefe do Grupo de Quadril do Instituto Nacional de Traumatologia e Ortopedia - INTO - Rio de Janeiro (RJ), Brasil.

3. Médico Ortopedista do Grupo de Quadril do Instituto Nacional de Traumatologia e Ortopedia - INTO - Rio de Janeiro (RJ), Brasil.

4. Médico Ortopedista, Estagiário do Grupo de Quadril do Instituto Nacional de Traumatologia e Ortopedia - INTO - Rio de Janeiro (RJ), Brasil.

Endereço para correspondência: Bruno Tavares Rabello, Rua Tonelero, 380-302, Copacabana - 22030-000 - Rio de Janeiro (RJ), Brasil. E-mail: brunotrabello1@yahoo.com.br

Recebido em 15/1/07. Aprovado para publicação em 27/8/08.

Copyright RBO2008 dos bons e excelentes foram observados em 22 pacientes, com destaque para a melhora do parâmetro dor. Não foi evidenciado qualquer caso de afrouxamento. Complicações foram observadas em dois casos, um paciente apresentou trombose venosa profunda (TVP), comprovada com doppler, e um caso de fratura peroperatória distal ao calcar, tratada com cerclagem, sem repercussão na qualidade dos resultados clínico e radiográfico do paciente. Conclusão: Os resultados sugerem, após seguimento médio de 5,6 anos, que a artroplastia total do quadril não cimentada pode ser opção satisfatória nas coxartroses de pacientes portadores de artrite reumatóide.

Descritores - Artrite reumatóide/radiografia; Artrite reumatóide/cirurgia; Artroplastia de quadril/métodos; Resultado de tratamento

\section{ABSTRACT}

Objective: To analyze clinical, functional, and radiographic results of uncemented total hip arthroplasties in patients with rheumatoid arthritis. Methods: Retrospective study of 24 patients (28 hips) with rheumatoid arthritis submitted to total hip arthroplasty, using uncemented femoral prosthesis Secur Fit Osteonics ${ }^{\circledR}$, with minimum follow-up of two years. The patients were clinically evaluated using the Merle D'Aubigné criteria and the Engh criteria in radiographic evaluation. Results: Mean follow-up time was 5.6 years (two years to eight years and 11 months). Clinically, good and excellent results were 
observed in 22 patients, with emphasis on pain relief. No case of loosening was seen. Two cases had complications: one patient had deep venous thrombosis (DVT), confirmed by doppler, and one case of fracture during surgery, distal to the calcar bone, treated with cerclage, with no repercussion in the quality of clinical and radiographic results of this patient. Conclusion: After a mean follow-up of 5.6 years, results show that uncemented total hip arthroplasty may be a satisfactory option in coxoarthroses of patients with rheumatoid arthritis.

\section{Keywords - Arthritis, rheumatoid/radiography; Arthritis, rheumatoid/surgery; Arthroplasty, replacement, hip/methods; Treatment outcome}

\section{INTRODUÇÃO}

A artroplastia total do quadril tornou-se excelente método de tratamento no alívio da dor e melhora funcional dos pacientes com doença degenerativa da articulação coxofemoral. Inicialmente sua indicação estava restrita a pacientes mais idosos e com demanda funcional menor; no entanto, o aperfeiçoamento da técnica cirúrgica, a evolução dos implantes e das superfícies de atrito, proporcionando menor desgaste, fizeram ampliar o universo dos pacientes que puderam se beneficiar com esse procedimento ${ }^{(1)}$.

Apesar da faixa etária mais jovem, a indicação de artroplastia de quadril em pacientes portadores de artrite reumatóide justifica-se pela dor e grave limitação da mobilidade causada pela afecção de base. Nesses pacientes, anormalidades na arquitetura e qualidade óssea são comuns e sua origem é multifatorial. A cavidade acetabular e a cabeça femoral normalmente encontram-se hipoplásicas. Protrusão acetabular e subluxação podem estar presentes. A metáfise é desproporcionalmente mais larga em relação ao canal femoral. Contratura de partes moles também faz parte desse espectro de alterações, principalmente dos tendões flexores, adutores e rotadores externos do quadril. Todos esses fatores implicam em experiência da equipe cirúrgica e planejamento pré-operatório adequado ${ }^{(2-3)}$.

Inicialmente, artroplastias cimentadas constituíam o padrão de escolha nesses pacientes, pois se acreditava que o componente cimentado se adaptaria melhor à complexa morfologia óssea. Trabalhos avaliando os resultados a longo prazo, entretanto, mostraram considerável percentual de sinais radiográficos de afrouxamento, impondo revisão tanto acetabular quanto femoral $^{(4-8)}$.

A baixa resistência do osso remodelado, a osteopenia e o aumento da atividade osteoclástica seriam a princípio pré-requisitos desfavoráveis às artroplastias não cimentadas. Na década de 80 , as artroplastias não cimentadas começaram a ganhar espaço, inicialmente tendo sua indicação formal nos pacientes jovens e sobretudo ativos ${ }^{(3)}$.

Cracchiolo et al analisaram retrospectivamente 34 pacientes submetidos à artroplastia total do quadril com implantes não cimentados, sem casos de afrouxamento após seguimento médio de 3,7 anos ${ }^{(9)}$. Resultados semelhantes foram relatados por Loehr et al e Kirk et al, comparando grupo de pacientes portadores de artrite reumatóide submetidos à artroplastias cimentadas e não cimentadas, não tendo os autores observado diferença estatística em relação à avaliação clínicofuncional ${ }^{(10-11)}$.

A proposta deste estudo é avaliar os resultados clínicos e radiográficos de 24 pacientes com artrite reumatóide submetidos à artroplastia total do quadril não cimentada pelo grupo de quadril do INTO.

\section{MÉTODOS}

No período compreendido entre fevereiro de 1996 e fevereiro de 2005, foram submetidos à artroplastia total do quadril não-cimentada, utilizando especificamente a prótese $S e c u r-F i t^{\circledR}, 29$ pacientes portadores de artrite reumatóide, totalizando 33 quadris. Foram excluídos do trabalho cinco pacientes, por apresentarem tempo de seguimento inferior a 24 meses, restando 24 pacientes, totalizando 28 quadris (quadro 1).

A haste femoral utilizada tem configuração de colo cônico, feita em liga de titânio e apresentando porosidade revestida em hidroxiapatita no terço proximal. A cúpula acetabular também apresenta revestimento em hidroxiapatita e cinco orifícios para colocação de parafusos.

A idade dos pacientes na época da cirurgia variou de 18 anos e um mês a 72 anos (média de 41,8 anos) 
QUADRO 1

Dados de identificação dos pacientes

\begin{tabular}{|c|c|c|c|c|c|c|c|}
\hline \multirow[t]{2}{*}{ Paciente } & \multirow[t]{2}{*}{$\begin{array}{l}\text { Idade na época } \\
\text { da cirurgia }\end{array}$} & \multirow[t]{2}{*}{ Sexo } & \multirow[t]{2}{*}{ Lado } & \multirow[t]{2}{*}{$\begin{array}{l}\text { Tempo de } \\
\text { seguimento }\end{array}$} & \multicolumn{2}{|c|}{$\begin{array}{l}\text { Avaliação } \\
\text { funcional }\end{array}$} & \multirow[t]{2}{*}{ Complicações } \\
\hline & & & & & Pré & Pós & \\
\hline 1 & 57 anos +8 meses & $\mathrm{F}$ & $\mathrm{D}$ & 4 anos +6 meses & 7 & 16 & \\
\hline 2 & 62 anos +2 meses & $\mathrm{F}$ & $\mathrm{D}$ & 6 anos +6 meses & 9 & 15 & \\
\hline 3 & 18 anos +1 mês & $\mathrm{F}$ & $\mathrm{D}$ & 4 anos +10 meses & 5 & 10 & \\
\hline 4 & 21 anos +4 meses & $\mathrm{F}$ & $\mathrm{D}$ & 8 anos +11 meses & 6 & 12 & \\
\hline 5 & 40 anos +5 meses & $\mathrm{M}$ & $E$ & 6 anos +11 meses & 10 & 16 & \\
\hline 6 & 41 anos + 1 mês & $M$ & $E$ & 5 anos +10 meses & 10 & 16 & \\
\hline 7 & 53 anos +8 meses & M & $\mathrm{D}$ & 6 anos +2 meses & 9 & 15 & \\
\hline 8 & 72 anos & $\mathrm{F}$ & $\mathrm{D}$ & 4 anos +9 meses & 8 & 15 & \\
\hline 9 & 68 anos +6 meses & $\mathrm{F}$ & $\mathrm{D}$ & 4 anos +6 meses & 9 & 14 & TVP \\
\hline 10 & 22 anos +10 meses & $\mathrm{F}$ & $\mathrm{D}$ & 3 anos +6 meses & 7 & 14 & \\
\hline 11 & $\begin{array}{l}22 \text { anos }+9 \text { meses } \\
23 \text { anos }+4 \text { meses }\end{array}$ & $\mathrm{F}$ & $\begin{array}{l}E \\
D\end{array}$ & $\begin{array}{l}5 \text { anos } \\
4 \text { anos }+5 \text { meses }\end{array}$ & $\begin{array}{l}8 \\
8\end{array}$ & $\begin{array}{l}16 \\
17\end{array}$ & \\
\hline 12 & 53 anos +1 mês & $\mathrm{F}$ & $\mathrm{D}$ & 2 anos +2 meses & 9 & 15 & \\
\hline 13 & 26 anos & $\mathrm{F}$ & $E$ & 3 anos +1 mês & 8 & 15 & \\
\hline 14 & 29 anos +7 meses & $\mathrm{F}$ & $E$ & 2 anos & 8 & 12 & \\
\hline 15 & 46 anos +4 meses & $\mathrm{F}$ & $E$ & 2 anos +1 mês & 10 & 15 & \\
\hline 16 & $\begin{array}{l}37 \text { anos }+2 \text { meses } \\
34 \text { anos }\end{array}$ & $\mathrm{F}$ & $\begin{array}{l}D \\
E\end{array}$ & $\begin{array}{l}3 \text { anos }+6 \text { meses } \\
6 \text { anos }+8 \text { meses }\end{array}$ & $\begin{array}{r}10 \\
9\end{array}$ & $\begin{array}{l}17 \\
16\end{array}$ & \\
\hline 17 & 28 anos +11 meses & $\mathrm{F}$ & $E$ & 4 anos +8 meses & 10 & 15 & \\
\hline 18 & 24 anos +2 meses & $\mathrm{F}$ & $E$ & 6 anos +11 meses & 9 & 15 & \\
\hline 19 & $\begin{array}{l}24 \text { anos }+6 \text { meses } \\
25 \text { anos }+4 \text { meses }\end{array}$ & $\mathrm{F}$ & $\begin{array}{l}D \\
E\end{array}$ & $\begin{array}{l}6 \text { anos }+8 \text { meses } \\
4 \text { anos }+6 \text { meses }\end{array}$ & $\begin{array}{l}8 \\
7\end{array}$ & $\begin{array}{l}14 \\
15\end{array}$ & \\
\hline 20 & 61 anos +1 mês & $F$ & $\mathrm{D}$ & 6 anos +2 meses & 6 & 15 & Fratura periprotética \\
\hline 21 & 45 anos +2 meses & $\mathrm{F}$ & $E$ & 8 anos +8 meses & 10 & 15 & \\
\hline 22 & 57 anos +3 meses & $\mathrm{F}$ & $\mathrm{D}$ & 8 anos +10 meses & 10 & 15 & \\
\hline 23 & 47 anos +2 meses & $\mathrm{F}$ & $\mathrm{D}$ & 8 anos +11 meses & 10 & 16 & \\
\hline 24 & $\begin{array}{l}48 \text { anos }+3 \text { meses } \\
49 \text { anos }+1 \text { mês }\end{array}$ & $\mathrm{F}$ & $\begin{array}{l}D \\
E\end{array}$ & $\begin{array}{l}6 \text { anos }+9 \text { meses } \\
5 \text { anos }+8 \text { meses }\end{array}$ & $\begin{array}{l}9 \\
9\end{array}$ & $\begin{array}{l}17 \\
15\end{array}$ & \\
\hline
\end{tabular}

F: Feminino; M: Masculino; D: Direito; E: Esquerdo; TVP: Trombose Venosa Profunda.

sendo 21 do sexo feminino e três do sexo masculino. $\mathrm{O}$ lado mais acometido foi o direito com 16 quadris e o lado esquerdo com 12 . O seguimento médio dos pacientes no ambulatório foi de 5,6 anos variando de dois anos a oito anos e 11 meses.

Todos os pacientes fizeram uso de, pelo menos, uma medicação no pré-operatório; antiinflamatório não esteróide (AINES) e/ou corticóide.

A técnica para colocação das próteses envolveu o planejamento pré-operatório visando determinar o ta- manho aproximado dos implantes, nível da osteotomia do colo femoral e possíveis deformidades que tivessem de ser corrigidas no ato operatório. O tamanho das próteses foi exatamente igual ao da última fresagem utilizada.

Todos os pacientes fizeram uso de antibioticoterapia profilática (cefazolina), iniciada na indução anestésica e mantida por 24 horas. Profilaxia da trombose venosa profunda (TVP) foi feita com heparina de baixo peso molecular na dose de $40 \mathrm{mg}$ via subcutânea, de 24 em 24 horas, por 10 dias. 
A via de acesso para o procedimento cirúrgico foi a ântero-lateral em 21 pacientes e a posterior em sete pacientes. Enxerto ósseo autólogo no acetábulo foi utilizado quando necessário, visando corrigir o centro de rotação do quadril nos casos de protrusão acetabular.

Os pacientes seguiram o protocolo do serviço de fisioterapia com carga parcial permitida na $6^{\mathrm{a}}$ semana de pós-operatório e carga total após 10 semanas de pós-operatório. O retorno ambulatorial seguiu o protocolo do grupo de quadril com acompanhamento clínico-radiográfico no primeiro, terceiro e sexto mês e, em seguida, anualmente.

$\mathrm{Na}$ consulta ambulatorial, todos os pacientes foram avaliados clinicamente com exame físico e radiográfico nas incidências em ântero-posterior e perfil do quadril, tanto no pré como no pós-operatório. Clinicamente, foram utilizados os critérios de Merle D’Aubigné et $a l^{(12)}$, cujos parâmetros consistem em dor, avaliação da marcha e arco de movimento, totalizando pontuação máxima de 18 (tabela 1).

TABELA 1

\begin{tabular}{|cc} 
Avaliação funcional pelos critérios de Merle D'Aubigné(12) \\
\hline Ruim & Menor que 12 \\
Razoável & $13-14$ \\
Bom & $15-16$ \\
Excelente & $17-18$
\end{tabular}

Fonte: Traduzido de: D'Aubigne RM, Postel M. Function al results of hip arthroplasty with acrylic prosthesis. J Bone Joint Surg Am. 1954;36-A(3):451-75.

$\mathrm{Na}$ análise radiográfica, foram avaliados sinais de instabilidade dos componentes: 1) critérios de fixação dos implantes (atrofia óssea proximal, traves ósseas, hipertrofia cortical) como descritos por Engh et $a l^{(13)}$; 2) a presença de osteólise de acordo com as áreas radiográficas de DeLee e Charnley(14) (figura 1) para o acetábulo e Gruen et al ${ }^{(15)}$ (figura 2) para o fêmur; 3) presença de ossificação heterotópica de acordo com a classificação de Brooker et $a l^{(16)}$.

Afrouxamento radiográfico foi definido como área de radiolucência progressiva maior do que $2 \mathrm{~mm}$ ou aumento da inclinação superior a $5^{\circ}$ para o componente acetabular e área de radiolucência de $2 \mathrm{~mm}$ ou mais em qualquer das zonas de Gruen et al ${ }^{(15)}$ para o componente femoral.

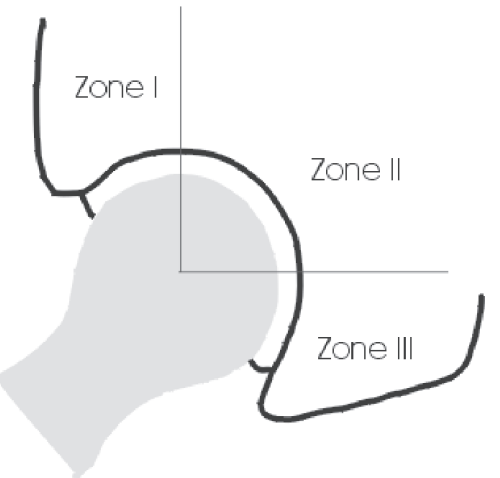

Figura 1 - Áreas radiográficas do acetábulo de DeLee e Charnley ${ }^{(14)}$

Fonte: DeLee JG, Charnley J. Radiological demarcation of cemented sockets in total hip replacement. Clin Orthop Relat Res. 1976;(121):20-32.

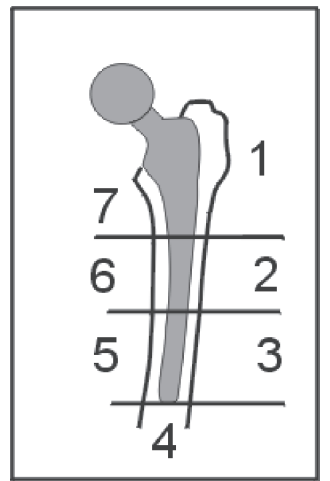

Figura 2 - Áreas radiográficas do fêmur de Gruen et al/(15)

Fonte: Gruen TA, McNeice GM Amstutz HC. "Modes of failure" of cemented stem-type femoral components: a radiographic analysis of loosening. Clin Orthop Relat Res. 1979;(141): 17-27.

\section{RESULTADOS}

Clinicamente, os pacientes foram avaliados pelos critérios de Merle D'Aubigné et al(12) que apresentaram melhora da média pré-operatória de 8,5 (5-10 pontos) para 14,8 pontos (10-17). Os resultados foram considerados bons e excelentes em 22 pacientes (gráfico $1)$.

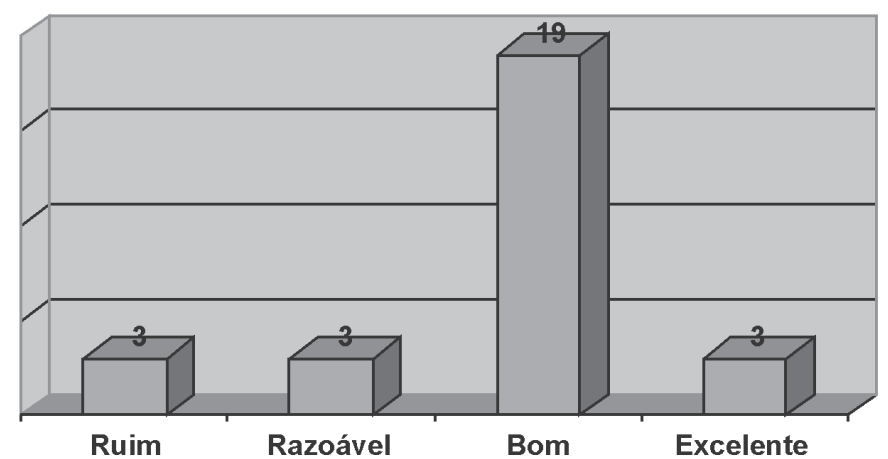

Gráfico 1 -Avaliação clínica dos pacientes pelos critérios de Merle D'Aubigné

A análise radiográfica demonstrou migração vertical do implante menor do que $3 \mathrm{~mm}$, aferido com paquímetro digital, em três casos. Sinais radiográficos de fixação do implante podem ser observados na tabela 2 e nas figuras 3, 4 e 5. Em quatro quadris identificamos pedestal na zona 4 de Gruen et $a l^{(15)}$. Nenhum 
TABELA 2

Avaliação radiográfica dos pacientes

\begin{tabular}{lcc}
\hline & Número de quadris & $\%$ \\
\hline Atrofia cortical & 19 & 67,8 \\
Traves ósseas & 11 & 39,2 \\
Migração vertical & 3 & 10,7 \\
Hipertrofia cortical & 13 & 46,4 \\
Pedestal & 4 & 14,2 \\
\hline
\end{tabular}
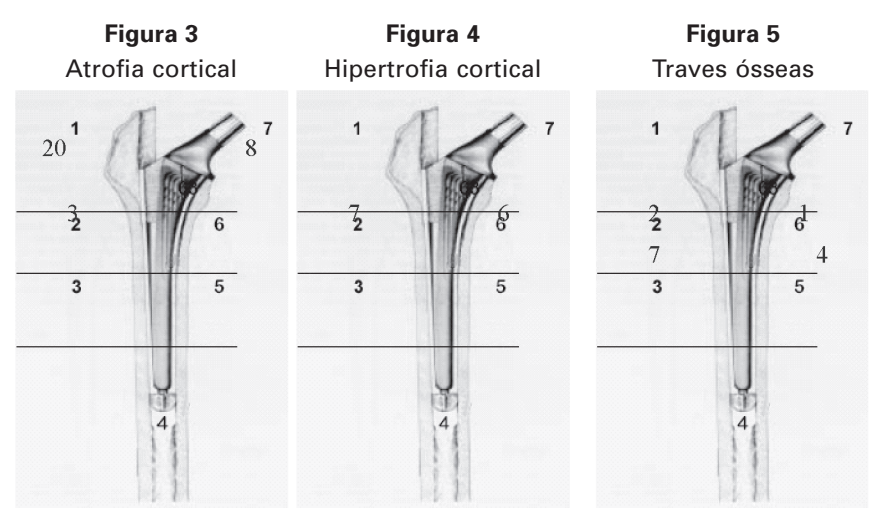

Figuras 3, 4, 5 - Número de casos de atrofia cortical, hipertrofia cortical e traves ósseas encontrados nas áreas radiográficas de Gruen

componente femoral apresentou sinais de radiolucência ao redor de toda circunferência do implante.

Os componentes femorais mais utilizados foram os de tamanho 6 e 7 (gráfico 2).

$\mathrm{Na}$ avaliação do acetábulo, quatro quadris apresentaram sinais de radiolucência de acordo com as zonas de DeLee e Charnley ${ }^{(14)}$, sendo três na zona I e II e um caso somente na zona II. Não houve migração de qualquer componente acetabular ou progressão da osteólise.

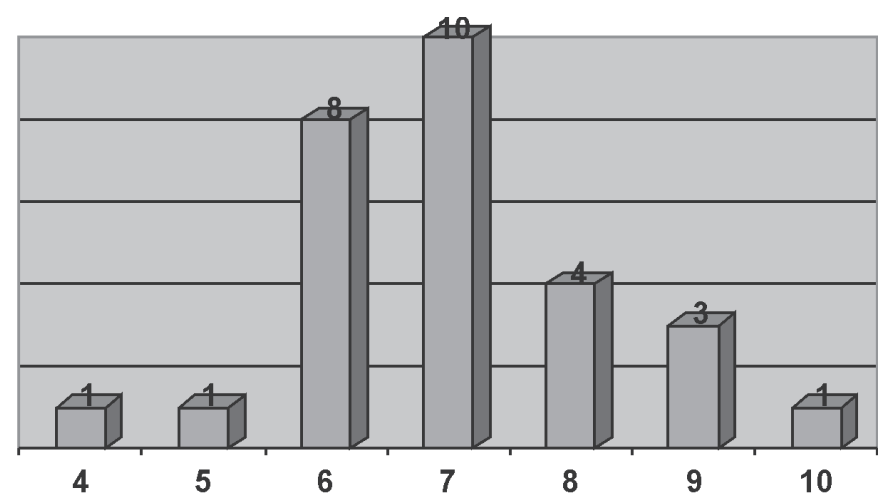

Gráfico 2 - Distribuição por tamanho dos componentes femorais

Rev Bras Ortop. 2008;43(8):336-42
Os componentes acetabulares mais utilizados foram os de tamanho 46, 48 e 50 (gráfico 3). Em 14 quadris não foi utilizado parafuso no acetábulo, em 10 quadris foi utilizado um parafuso e em quatro quadris dois parafusos foram utilizados (gráfico 4).

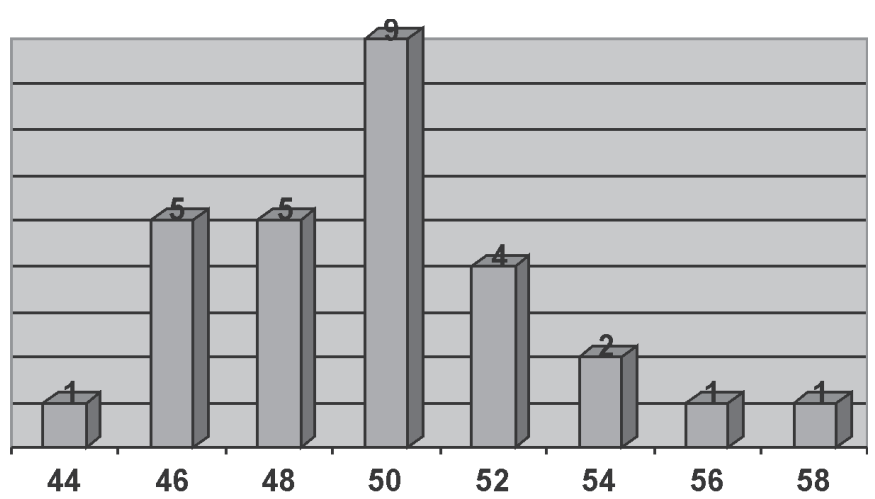

Gráfico 3 - Distribuição por tamanho dos componentes acetabulares

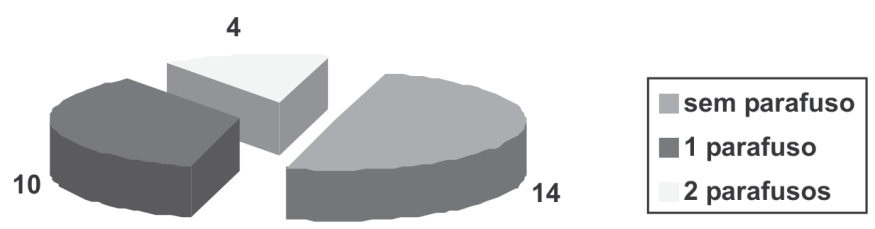

Gráfico 4 - Distribuição por número de parafusos acetabulares utilizados

Ossificação heterotópica foi evidenciada em dois casos, classificada de acordo com Brooker et al ${ }^{(16)}$, sendo em um caso tipo I e no outro tipo II.

Como complicações, um paciente evoluiu com trombose venosa profunda na perna ipsilateral na panturrilha no pós-operatório, confirmado com exame doppler e tratado clinicamente e outro paciente apresentou fratura periprotética distal ao calcar, identificada no peroperatório e tratada com cerclagem. Não houve migração vertical da haste ou afrouxamento do implante. Não houve casos de luxação ou infecção.

\section{DISCUSSÃO}

As artroplastias totais do quadril tornaram-se forma de tratamento satisfatório na melhora da dor e função dos quadris artríticos. Os pacientes com artrite reumatóide apresentam alterações peculiares na qualidade 
óssea tanto femoral quanto acetabular. A solução deste problema a princípio seria a utilização de cimento ósseo nas artroplastias. No entanto, trabalhos com objetivo de avaliar a evolução clínica e radiológica desses doentes demonstraram índices significativos de afrouxamento dos componentes, apesar da melhora funcional a longo prazo apresentada pelo grupo estudado $^{(4-8)}$.

Clinicamente, nossos pacientes mostraram melhora considerável de 8,5 para 14,8 pontos utilizando os critérios de Merle D’Aubigné et al ${ }^{(12)}$. Dor foi a variável que demonstrou melhora mais expressiva, os pacientes evoluíram com média de 2 para 5 no pós-operatório. Obtivemos resultados bons e excelentes em 78,5\% dos casos. Resultados ruins foram encontrados em três pacientes, sendo que em um dos casos, apesar da melhora da dor, a presença de alterações em outras articulações limitava a marcha e, mesmo após a cirurgia, este paciente permaneceu restrito à marcha com duas muletas.

Esses valores, entretanto, são relatados tanto nas artroplastias não cimentadas quanto nas cimentadas ${ }^{(11)}$. Acreditamos que a mudança nesses valores só poderá ser avaliada com o acompanhamento desses pacientes, comparando-se as duas diferentes técnicas de fixação.

Vários estudos comprovaram que o afrouxamento asséptico dos componentes de artroplastias cimentadas do quadril ocorre em pacientes com artrite reumatóide, apesar da baixa demanda funcional por eles apresentada $^{(2-8)}$. Essa constatação direcionou os estudos para avaliação do resultado de artroplastias de quadril não cimentadas nestes pacientes.

Poss et al, utilizando artroplastias totais cimentadas, relataram que $78 \%$ dos pacientes apresentavam radiolucência progressiva no acetábulo e em cinco casos foi indicada revisão femoral ${ }^{(6)}$. Severt et al após avaliar 75 pacientes, relataram 5,3\% de afrouxamento do componente femoral e 9,3\% do componente acetabu$\operatorname{lar}^{(5)}$. Resultados similares foram encontrados por Unger et $a l^{(7)}$ e Lachiewicz et $a l^{(4)}$. Learmonth et al relataram taxa de afrouxamento de $57 \%$ dos implantes ${ }^{(8)}$.

Na revisão bibliográfica das artroplastias não cimentadas, Loehr et al relataram 21 casos, com seguimento médio de 3,3 anos, evidenciando $43 \%$ de áreas pequenas de radiolucência nos componentes femorais e $12,8 \%$ nos componentes acetabulares ${ }^{(10)}$. Nenhum componente necessitou de revisão. Cracchiolo et al descreveram os resultados de 40 artroplastias não cimentadas sem casos de afrouxamento após tempo de seguimento médio de 3,7 anos ${ }^{(9)}$. Resultados similares foram publicados por Effenberger et $a l^{(17)}$, $\operatorname{Lord}^{(18)} \mathrm{e}$ Chandler et $a l^{(19)}$.

Nos 24 pacientes avaliados no nosso trabalho, observamos: 1) no componente acetabular, quatro casos apresentaram linhas radiolucentes, sem progressão radiográfica ou sinais de instabilidade do implante; 2) não detectamos este achado radiográfico na avaliação femoral. Cracchiolo et al relataram que $43 \%$ dos componentes femorais e 12,8\% dos acetábulos apresentavam áreas de radiolucência, mas em nenhum caso envolviam toda a circunferência da interface próteseosso $^{(9)}$. Valores superiores foram relatados por Effenberger et $l^{(17)}$ e Loehr et $l^{(10)}$, mas sem relato de revisão dos implantes.

Sinais radiográficos de fixação dos componentes descritos por Engh et al ${ }^{(13)}$, como a hipertrofia cortical, foram notados em 13 quadris, principalmente nas zonas III e V, justamente onde a haste não apresenta porosidade e sua presença pode ser explicada pela transferência de forças. Effenberger et al ${ }^{(17)}$ relataram esse sinal em $60 \%$ dos pacientes e Cracchiolo et al $l^{(9)}$ em $12,5 \%$ dos casos.

A presença de traves ósseas ou reação óssea endosteal foi observada em 11 quadris. Cracchiolo et al relataram tal sinal em $43 \%$ dos $\operatorname{casos}^{(9)}$. Esses valores estão distantes dos descritos por Engh et al em pacientes não reumatóides ${ }^{(20)}$. Atrofia óssea proximal foi observada em 19 quadris $(67,8 \%)$ principalmente nas zonas I e VI de Gruen et al ${ }^{(15)}$, localização similar foi encontrada por Loehr et al ${ }^{(10)}$. Effenberger et al relataram que $40 \%$ dos 70 pacientes apresentavam atrofia óssea proximal ${ }^{(17)}$. Outros trabalhos relatam valores que variam de $23-100 \%{ }^{(9,11,18-19)}$ dos casos. Esses achados geraram certa preocupação em alguns autores, pois sua progressão radiográfica poderia definir afrouxamento, principalmente naqueles pacientes na $2^{\mathrm{a}}$ década de 
$\operatorname{artroplastia~}^{(21-22)}$. Acreditamos que somente estudos a longo prazo podem dirimir essa dúvida.

\section{CONCLUSÃO}

De acordo com os resultados apresentados, acreditamos que as artroplastias totais de quadril não cimentadas em pacientes portadores de artrite reumatóide apresentam bom resultado clínico e radiológico após seguimento médio de 5,6 anos, o que não difere dos resultados encontrados em trabalhos em que o número de pacientes e o tempo de acompanhamento são maiores.

\section{REFERÊNCIAS}

1. Canale ST, editor. Campbell's operative orthopaedics. 10a ed. St. Louis: Mosby; 2003.

2. Callaghan JJ, Rosenberg AG, Rubash HE, editors. The adult hip. Philadelphia: Lipppincott-Raven; 1997.

3. Haber D, Goodman SB. Total hip arthroplasty in juvenile chronic arthritis: a consecutive series. J Arthroplasty. 1998; 13(3):259-65.

4. Lachiewicz PF, McCaskill B, Inglis A, Ranawat CS, Rosenstein BD. Total hip arthroplasty in juvenile rheumatoid arthritis. Two to eleven-year results. J Bone Joint Surg Am. 1986;68(4):5028.

5. Severt R, Wood R, Cracchiolo A 3rd, Amstutz HC. Long-term follow-up of cemented total hip arthroplasty in rheumatoid arthritis. Clin Orthop Relat Res. 1991;(265):137-45.

6. Poss R, Maloney JP, Ewald FC, Thomas WH, Batte NJ, Hartness C, Sledge CB. Six-to 11-year results of total hip arthroplasty in rheumatoid arthritis. Clin Orthop Relat Res. 1984;(182):109-16.

7. Unger AS, Inglis AE, Ranawat CS, Johanson NA. Total hip arthroplasty in rheumatoid arthritis. A long-term follow-up study. J Arthroplasty. 1987;2(3):191-7.

8. Learmonth ID, Heywood AW, Kaye J, Dall D. Radiological loosening after cemented hip replacement for juvenile chronic arthritis. J Bone Joint Surg Br. 1989;71(2):209-12.

9. Cracchiolo A 3rd, Severt R, Moreland J. Uncemented total hip arthroplasty in rheumatoid arthritis disease. A two-to six-year follow-up study. Clin Orthop Relat Res. 1992;(277):166-74.
10. Loehr JF, Munzinger UD, Tibesku C. Uncemented total hip arthroplasty in patients with rheumatoid arthritis. Clin Orthop Relat Res. 1999;(366):31-8.

11. Kirk PG, Rorabeck CH, Bourne RB, Burkart B. Total hip arthroplasty in rheumatoid arthritis: comparison of cemented and uncemented implants. Can J Surg. 1993;36(3):229-32. Comment in: Can J Surg. 1993;36(3):199.

12. D'Aubigne RM, Postel M. Function al results of hip arthroplasty with acrylic prosthesis. J Bone Joint Surg Am. 1954;36-A(3):451-75.

13. Engh CA, Massin P, Suthers KE. Roentgenographic assessment of the biologic fixation of porous-surfaced femoral components. Clin Orthop Relat Res. 1990;(257):107-28. Erratum in: Clin Orthop. 1992;(284):310-2. Comment in: Clin Orthop Relat Res. 1992;(284):310-2.

14. DeLee JG, Charnley J. Radiological demarcation of cemented sockets in total hip replacement. Clin Orthop Relat Res. 1976; (121):20-32.

15. Gruen TA, McNeice GM, Amstutz HC. "Modes of failure" of cemented stem-type femoral components: a radiographic analysis of loosening. Clin Orthop Relat Res. 1979;(141):1727.

16. Brooker AF, Bowerman JW, Robinson RA, Riley LH Jr. Ectopic ossification following total hip replacement. Incidence and a method of classification. J Bone Joint Surg Am. 1973; 55(8):1629-32.

17. Effenberger H, Ramsauer T, Böhm G, Hilzensauer G, Dorn U, Lintner F. Successful hip arthroplasty using cementless titanium implants in rheumatoid arthritis. Arch Orthop Trauma Surg. 2002;122(2):80-7.

18. Lord GA. Madreporique stemmed total hip replacement: five years' clinical experience. J R Soc Med. 1982;75(3):66-76.

19. Chandler HP, Reineck FT, Wixson RL, McCarthy JC. Total hip replacement in patients younger than thirty years old. A fiveyear follow-up study. J Bone Joint Surg Am. 1981;63(9):142634.

20. Engh CA, Massin P. Cementless total hip arthroplasty using the anatomic medullary locking stem. Results using a survivorship analysis. Clin Orthop Relat Res. 1989;(249):141-58.

21. Bugbee WD, Culpepper WJ 2nd, Engh CA Jr, Engh CA Sr. Long-term clinical consequences of stress-shielding after total hip arthroplasty without cement. J Bone Joint Surg Am. 1997; 79(7):1007-12.

22. Tanzer M, Malloney WJ, Jasty M, Harris WH. The progression of femoral cortical osteolysis in association with total hip arthroplasty without cement. J Bone Joint Surg Am. 1992; 74(3):404-10. 